\title{
Modelos de calidad aplicados al servicio de mediación en México ${ }^{1}$ \\ Quality models applied to the mediation services in Mexico
}

Roberto N., Guerrero-Vega $^{2}$
Universidad Politécnica de Nicaragua, Nicaragua
(iD) https://orcid.org/0000-0003-3847-0326
doi) https://doi.org/10.29105/pgc6.12-8

RESUMEN

El presente artículo es una investigación que posee un enfoque meramente cualitativo, parte de un diseño no experimental, bajo un nivel documental-bibliográfico trasversal. Tiene por objetivo general analizar los principales modelos de evaluación de la calidad y definir cuál de ellos ha sido aplicado para evaluar el servicio de mediación en México. Tras la revisión de la literatura y el análisis documental se determinó que: Los principales modelos de calidad de índole nacional e internacional, aún no han sido aplicados para evaluar el servicio mediación, ya que están enfocados en evaluar un contexto general como lo es el sistema de justicia tradicional y otros rubros relacionados a la comercialización de productos y servicios. Sin embargo, se determinó que la calidad del servicio de mediación ha sido evaluada por modelos estandarizados, desarrollados por algunas investigaciones académicas. Se concluye que en México existe la necesidad de implementar modelos rigurosos y estandarizados encargados de evaluar al servicio de mediación, con la finalidad de obtener una certificación nacional e internacional que ratifique a las instituciones que cumplen con los estándares de calidad.

Palabras Clave: Calidad, consumidor, mediación, modelos, servicio.

\section{ABSTRACT}

This article is a research that has a purely qualitative approach, part of a non-experimental design, under a cross-sectional documentary-bibliographic level. Its general objective is to analyze the main quality evaluation models and to define which of them has been applied to evaluate the mediation service in Mexico. After reviewing the literature and the documentary analysis it was determined that: The main national and international quality models have not yet been applied to evaluate the mediation service, since they are focused on assessing a general context such as the traditional justice system and other items related to the marketing of products and services. However, it was determined that the quality of the mediation service has been evaluated by standardized models developed by some academic research. It is concluded that in Mexico there is a need to implement rigorous and standardized models to evaluate the mediation service, with the aim of obtaining a national and international certification that ratifies the institutions that meet the quality standards.

Keywords: Consumer, mediation, models, quality, service.

Recibido: 26 de Noviembre 2019 - Aceptado: 21 de Abril 2020 - Corregido: 11 de Mayo 2020

Cómo referenciar este artículo:

Guerrero-Vega, R. N. (2020). Modelos de calidad aplicados al servicio de mediación en México. Politica Globalidad y Ciudadania, 149-169. Recuperado de http://revpoliticas.uanl.mx/index.php/RPGyC/article/ view/152

\footnotetext{
1 Artículo de revisión derivado del proyecto de investigación: La eficacia del mediador como factor determinante en la calidad del proceso de mediación.

2 Doctorando en Métodos Alternos de Solución de Conflictos, Universidad Autónoma de Nuevo León. Docente Investigador, Universidad Politécnica de Nicaragua. Email: neftali2787@gmail.com. Orcid ID: orcid.org/0000-0003-3847-0326.
} 


\section{1.- INTRODUCCION}

Las primeras expresiones históricas de la calidad en el ámbito industrial surgieron a mediados de los años 70s, cuando las grandes compañías dedicadas a la manufactura en el mundo, empezaron a competir con las corporaciones norteamericanas. Estas compañías fueron pioneras en cambiar sus filosofías operativas, entre los primeros enfoques se aplicó la disminución del tamaño de las industrias, trayendo consigo cambios sustanciales orientados a la satisfacción de los clientes y el perfeccionamiento de las comunicaciones dentro de las compañías (Diaz, 2016).

Los retos planteados por las compañías en relación a la calidad estaban enmarcados en promover y aumentar la productividad, la capacidad de respuesta, la tecnología, comunicaciones, servicio al cliente y la reducción de costos.

La calidad no solo se ha manifestado en el ámbito industrial, también se ha venido reflejando en diferentes civilizaciones como, por ejemplo: En la antigua Grecia donde las primeras poblaciones le denominaban arete, término que era entendido como excelencia (Barraza, 2007). Por otra parte, en la era del antiguo Egipto ya existía la figura del inspector de calidad, quien era el encargado de verificar las dimensiones y simetría de los bloques que posteriormente eran utilizados en las grandes construcciones que edificaba esta civilización. También en China el antiguo Sun Tzu implementaba una serie de estrategias dirigidas a mejorar la calidad del entrenamiento de sus soldados y también implementaba la calidad en sus técnicas de guerra (Sun, 2009).

En el siglo XVII y XVIII emanan en las grandes ciudades los gremios de artesanos, quienes establecen especificaciones y requisitos para los materiales con los que se trabajaban y definen procesos y productos para su elaboración. Estas acciones son consideradas como el inicio de las organizaciones de certificación, debido a que estos gremios llevaban un control específico de admisión de sus asociados de acuerdo a los requerimientos establecidos, este factor les permitía mantener la calidad de su trabajo y de sus miembros (Miranda Gonzalez, 2007).

Etimológicamente la calidad es de procedencia latina, y es denominada como "clase" o "tipo"; esta definición promueve diferentes interpretaciones por su abstracta expresión. En la actualidad para la Real Academia Española, la calidad es definida como "El conjunto de propiedades inherentes a una cosa que permite apreciarla como igual, mejor o peor de las restantes de su especie" (RAE, 2019).

Existen otras definiciones que abordan a la calidad desde sus diferentes perspectivas, por ejemplo:

Para Drucker (1990) la calidad no es lo que contiene el servicio, es lo que el cliente obtiene

Revista Política, Globalidad y Ciudadanía, Vol. 6. No. 12, Julio - Diciembre 2020, Universidad Autónoma de Nuevo León, Monterrey, México, ISSN 2395-8448. 149-169. http://revpoliticas.uanl.mx/index.php/RPGyC/article/view/152 
de él y por lo que está dispuesto a pagar (pág. 41); en cambio Ishikawa (1986) plantea a la calidad enfocada en el producto sin menoscabar la utilidad de la calidad del servicio, trabajo de calidad, calidad del proceso y cualquier otro enfoque relacionado a la excelencia.

Lechuga et al (2017) La calidad es una de las características clave de los productos y servicios que se mide nacional e internacionalmente a través de las denominadas empresas certificadoras de calidad. Las apariciones de estas certificaciones surgen por la necesidad de homogenizar la medición de la calidad de los productos y servicios. (p.34).

Por otro lado, hay que resaltar que la calidad no solo se refiere a la finalización del producto o del servicio, también está enfocada a la calidad del proceso que esencialmente es el causante de la excelencia de los anteriores factores (Imai, 1998, pág. 10). De acuerdo a lo anterior se puede afirmar que con el paso del tiempo el concepto de calidad ha evolucionado su enfoque, ya no solo se encuentra relacionado en aumentar la productividad o en ofertar un mejor servicio al cliente; esta definición de calidad es más amplia, así lo refiere (Diaz, 2016) quien también plantea:

"La calidad debe entenderse y debe de ser transmitida como un valor que genera actitudes y comportamientos en el trabajo y en la vida privada del trabajador; también se trata de buscar conscientemente los máximos estándares deseables en todo lo que realizamos en la vida; es una filosofía que debe de estar detrás de todos los movimientos del individuo; es un estilo de vida; es una cultura, donde lo principal es el trabajo, el servicio, la entrega completa" (pág. 128).

Es notable que las referidas definiciones están planteadas con un enfoque dirigido al producto, servicio ofertado o las fases del proceso que conlleva a la calidad, cabe señalar que todas las concepciones convergen un denominador común, el cual es construir y alcanzar estándares de excelencia.

En México existen diferentes modelos nacionales e internacionales que evalúan la calidad de los servicios y productos que ofertan las diferentes instituciones. Entre los más relevantes están el Premio Nacional de Calidad (PNC), la Organización Internacional para la Estandarización (ISO). Estos modelos en su mayoría están dirigidos a evaluar actividades relacionadas a la industria, sin embargo, esta filosofía de calidad ha trascendido a otros campos como los son la salud, la justicia, la educación entre otros.

Por tanto, en el presente trabajo además de analizar los principales modelos de evaluación de calidad, también se desarrolla cuáles de estos modelos se han implementado en algunos rubros en México, al igual se resalta como problemática que estos sistemas de evaluación aún 
no han sido aplicados en los Centros públicos o privados que se dedican a ofertan el servicio de mediación en México.

Con la finalidad de proponer variantes o posibles soluciones al fenómeno que antecede, en este estudio se menciona una serie de investigaciones académicas que han evaluado el servicio de mediación desde diferentes perspectivas, partiendo de modelos estandarizados de calidad.

\section{2.- FUNDAMENTO TEÓRICO}

\section{La prestación del servicio de mediación}

Un servicio representa un conjunto de acciones encaminadas a servir en algo o a alguien, estas acciones tienen por finalidad cumplir con la satisfacción del individuo o individuos que los requieren.

La Ley de mecanismos alternativos para la solución de controversias en el estado de Nuevo León (2017), define a los sujetos e intuiciones encargados en prestar el servicio de mediación. En primer lugar se encuentra el Instituto de Mecanismos Alternativos para la Solución de Controversias, quien es el órgano del Consejo de Judicatura, que regula y presta los servicios en materia de los mecanismos alternativos de solución de conflictos (MASC); seguido están los Centros de mecanismos alternativos, que son definidos por la ley como "Todas las instituciones públicas y privadas que brindan servicios de mecanismos alternativos, distintas al Instituto“, cabe destacar que dichos centros deben contar con la acreditación del instituto.

Por otro lado, también la ley define al facilitador o mediador como la persona física que cuenta con certificación o certificaciones especializadas respaldadas por el Instituto, en virtud de prestar los servicios de los MASC.

De acuerdo a estas definiciones establecidas por la norma, no cabe duda que la mediación es considerada un servicio y el mediador es la persona encargada de materializarlo. De igual manera es importante destacar que la asistencia que funge el mediador no solo coincide con la definición de servicio, también se relaciona con las características generales de los servicios. De acuerdo a Zeimthaml y Berry (1988), las características consisten en la intangibilidad, la heteromogeneidad, la inseparabilidad y el carácter perecedero. En el siguiente apartado se desarrollarán las referidas características partiendo de su conceptualización y la relación que existe con el servicio de la mediación:

\section{La Intangibilidad}

Para Lovelock (1983) todos los servicios son considerados intangibles debido a que no se trata de un objeto o producto más bien son resultados, así mismo plantea que los servicios no podrán ser juzgado por el consumidor antes de adquirirlos, en vista que estos no pueden sentirse, 
palparse, verse o tocarse. Es por ello que el servicio de mediación, se identifica con la intangibilidad ya que no se trata de un bien o producto del cual se pueda tener posesión; además su valoración no puede ser en base a sus características físicas ya que son inexistentes. El servicio mediación y su intangibilidad puede ser evaluada en base a la percepción e interpretación del usuario o cliente.

\section{La heteromogeneidad}

Esta característica se basa en la variabilidad del servicio ofertado y los resultados obtenidos, estos resultados no pueden ser medibles de forma estandarizada, ya que cada consumidor y el servicio ofertado son diferentes (Duque Oliva, 2005). Partiendo de lo anterior se debe considerar que el servicio de mediación siempre va ser variable ya que ningún proceso de mediación resulta ser igual a otro; esta variabilidad dependerá del actuar de las partes en el proceso de mediación o de las habilidades que aplique el tercero neutral al gestionar el conflicto.

\section{La inseparabilidad}

De acuerdo a Duque (2005) este factor está relacionado con la simultaneidad, se trata de la interacción que hay entre la persona prestadora del servicio y el usuario o cliente que lo consume. Esta característica está centrada en el consumo, factor que será base de la distinción entre el producto y servicio. El producto se crea se vende y posteriormente se consume por otra parte el servicio se oferta y se consume en un mismo instante. En cuanto a la mediación como servicio sucede exactamente el mismo debido a que se oferta y se consume en el momento de su aplicación.

\section{De carácter perecedero}

Esto significa que los servicios no subsisten en el tiempo, algunos pueden ser de un solo momento en cambio otros pueden efectuarse en diferentes etapas, partiendo de esta lógica queda claro que al no tratarse de un producto el servicio no podrá ser almacenado (Diaz, 2016). El servicio de mediación ofertado por las instituciones descritas en la parte inicial de este acápite, se clasifica de carácter perecedero, debido a que no es tangible y en consecuencia no puede ser acopiado, de acuerdo a esta situación se garantiza la imperdurabilidad del servicio ofertado. Este servicio persistirá solo durante tiempo que dure el proceso de mediación y también durante se promulguen las diferentes sesiones próximas.

Al leer lo anterior resulta imprescindible no establecer las diferencias que existen entre las características que contienen un producto y un servicio por ello se plantea el siguiente cuadro: 


\section{Cuadro 1. Diferencias entre Producto y Servicio}

\begin{tabular}{|c|c|}
\hline Producto & Servicio \\
\hline $\begin{array}{l}\text { - Es tangible y palpable } \\
\text { - Un producto podrá ser idéntico a otro } \\
\text { - El producto se crea se vende y posterior- } \\
\text { mente se consume } \\
\text { - El producto de acuerdo a su tipología } \\
\text { puede perecer o descomponerse }\end{array}$ & $\begin{array}{l}\text { - Intangible y no pueden ser evaluado por } \\
\text { su aspecto físico } \\
\text { - El servicio ofertado al consumidor siem- } \\
\text { pre va ser variable } \\
\text { - El servicio se oferta y se consume en un } \\
\text { mismo instante } \\
\text { - No persiste en el tiempo ni puede ser al- } \\
\text { macenado }\end{array}$ \\
\hline
\end{tabular}

Fuente: Elaboración propia, 2020

De acuerdo a estas diferencias y a las características antes planteadas queda claro que existe una relación amplia entre el servicio de mediación y los preceptos generales del servicio, por lo tanto, la medición como un servicio puede estar sujeta a cualquier tipo de evaluación, siempre y cuando esta se aplique con la perspectiva de mejorar la calidad de los procesos inmersos en el servicio.

\section{Modelos de evaluación de calidad}

Dando continuidad al enfoque de calidad, es pertinente mencionar que existen diferentes modelos dedicados a gestionar la calidad desde sus diferentes perspectivas. Estos modelos sirven de guías para todas las instituciones privadas o públicas que se dedican a mejorar un producto $\mathrm{u}$ ofrecer un servicio.

Históricamente en diferentes países del mundo han existido esfuerzos en mejorar los procesos de calidad de las empresas, partiendo de la dinámica de la evaluación y el reconocimiento. En el año 1951, Japón instituyo el premio Deming como reconocimiento a las empresas que alcanzaran mejorar sus procesos de calidad; por otro lado, en los Estados Unidos se otorgaba la distinción Malcon Baldrige, que concordaba con el mismo enfoque de calidad. En el año 1989 México creo el premio nacional de calidad, este era entregado por el presidente de la republica a las empresas que cumplían con el modelo nacional de competitividad, cabe destacar que el proceso de selección y valoración a las instituciones estaba a cargo del Instituto para Fomento a la Calidad Total A.C (Diaz, 2016).

Existen otros modelos de gestión de calidad que se encuentran ligados a la normalización y estandarización. Para hablar de ellos primero se debe de tener una visión clara de los referidos enfoques. Castañeda Salas (2019) en su investigación cita diferentes autores que definen estos dos enfoques: 


\section{Normalización}

Según Merlo Vega (2011) la normalización se define .... como la guía que permite definir como debe ser un producto o servicio, de manera que todas las organizaciones que lo desarrollen sigan un mismo modelo... con ello se consigue... que estos productos y servicios cumplan con uno de los criterios mínimos para que puedan ser evaluados y comparados para establecer un nivel de calidad determinado, por otra parte asegura que los productos y servicios sean compatibles entre si

\section{Estandarización}

Se define como "... aquellos criterios por los que puede medirse y evaluarse los servicios... establecidos por las organizaciones profesionales, corporaciones acreditadas u organismos estatales, los criterios pueden reflejar, los diversos modos o un mínimo ideal un procedimiento modélico, una medida cuantitativa o una evaluación cualitativa" (Merlo Vega, 2011)

Los modelos de normalización y estandarización para algunos autores o instituciones son utilizados como sinónimos, pero de acuerdo a las definiciones antes planteadas poseen diferentes características; en este sentido y con el ánimo de diferenciar ambos modelos a continuación se plantea un cuadro ilustrativo.

\section{Cuadro 2. Diferencias entre Normalización y Estandarización.}

\begin{tabular}{|c|c|}
\hline Normalización & Estandarización \\
\hline 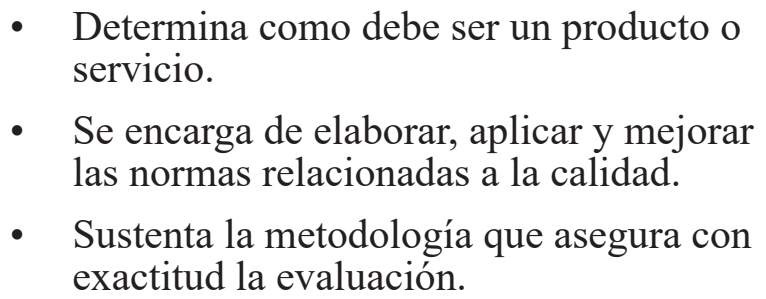 & $\begin{array}{l}\text { Define características y criterios para que } \\
\text { un producto o servicio pueda ser suscep- } \\
\text { tible de medición o evaluación. } \\
\text { - Desarrolla sistemáticamente la actualiza- } \\
\text { ción de los patrones, medidas y especifi- } \\
\text { caciones de los productos o servicios. }\end{array}$ \\
\hline
\end{tabular}
Fuente: (Tafolla, 2000) (SE, 2016); (Castañeda Salas, 2019) elaboración propia, (2020).

Las características de la normalización y la estandarización son parámetros establecidos con el fin de mejorar la calidad de los procesos en los diferentes ámbitos e instituciones.

Sistema organización internacional para la estandarización (ISO)

Después de la segunda guerra mundial las Naciones Unidas en 1947 crean la Organización Internacional de Normalización (ISO), que tiene como propósito promover normas y regula- 
ciones internacionales para la fabricación de los productos en donde se garantice su calidad y seguridad, posterior en 1951 se publicó el primer estándar ISO dirigido a una referencia de temperatura que se encargaba de medir las longitudes dentro de la industria, siguiendo esta secuencia, ISO creo todo tipo de normas enfocadas en productos, servicios y procesos relacionados al ámbito de la vida (Castañeda Salas, 2019)

En la actualidad 157 países son parte de la red de organizaciones internacionales de normalización, estas instituciones operan en países grandes o pequeños, industrializados o en vías de desarrollo de todas las regiones del mundo (ONUDI, 2010). Una peculiaridad de estas organizaciones es que, a pesar de tener un gran impacto en el ámbito industrial y empresarial, su naturaleza yace en el voluntariado, esto quiere decir que estas normas no pueden ser impuestas de maneras coercitivas para las instituciones públicas o privadas al menos que estás los soliciten.

De acuerdo a lo que plantea Aenor (2002) ISO despliega una serie de normas técnicas de carácter voluntario que incrementan el valor a todas las operaciones empresariales, entre estas virtudes se encuentran:

La contribución y el desarrollo de la fabricación y suministro de productos y servicios más eficientes, seguros y limpios.

Brinda la protección a consumidores con respecto a los productos y servicios.

Facilita y ajusta el comercio entre países.

Mejora la satisfacción de la clientela.

Genera confianza en los consumidores o usuarios.

Se obtiene una certificación de índole internacional.

Es importante observar que entre los beneficios que ofrece la aplicación y el seguimiento de las normas ISO, se pueden destacar tres elementos fundamentales que se han venido planteando en cuanto a la calidad del proceso de mediación, entre ellos están la satisfacción al cliente, la generación de confianza entre los usuarios de cara a las instituciones y por último la calidad partiendo de un servicio más eficaz.

Existe una variedad de normas ISO que han sido creadas a partir de la reaparición de nuevos productos o servicios ofertados a la sociedad. En la actualidad no se sabe con exactitud cuántos tipos de las normas ISO existen, debido a que estás siempre están en constante cambio, es por ello que en el siguiente grafico se reflejaran las normas más influyentes de algunos ámbitos: 
Gráficas 1. Tipos de normas ISO

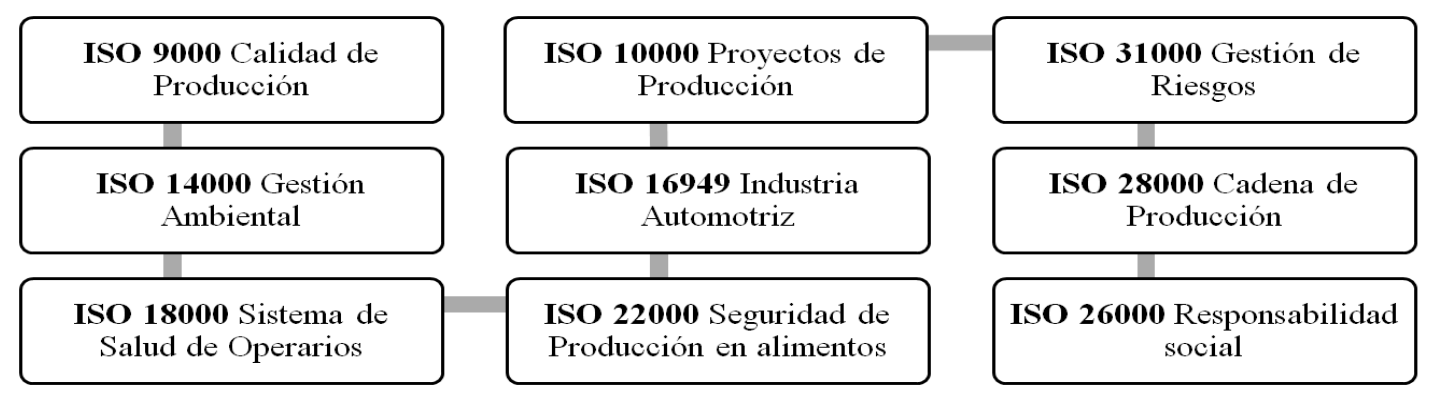

Fuente: Elaboración propia, (2020).

En la referida tipología se destaca la ISO 9000, esta norma se encuentra orientada a la estandarización de los sistemas de calidad que no están relacionadas con algún producto, sino con los procesos de los que derivan los productos y servicios. La finalidad de esta norma es emplear los métodos, técnicas y procedimientos específicos relacionados al cumplimiento de principios, objetivos y metas destinados a satisfacer las necesidades de los consumidores o usuarios (Delgado, 2011).

La certificación de norma ISO 9000 y la 9001 son acreditaciones muy demandadas por las diferentes instituciones privadas y públicas dedicadas a ofrecer un producto o brindar un servicio. En lo que concierne a México específicamente al estado de Nuevo León, las entidades que lograron alcanzar esta certificación son: el Tribunal Superior de Justicia y otras jurisdicciones u órganos judiciales y la Procuraduría Federal del Consumidor (PROFECO).

\section{Norma ISO 9001 adoptada por el Tribunal Superior de Justicia del Estado de Nuevo León}

El Tribunal Superior de Justicia del Estado de Nuevo León (2003) público en su informe trimestral número 7, algunos de sus principales logros en donde destaco la acreditación ISO 90012000 del Tribunal Superior de Justicia y la postulación de un pilotaje de otras áreas jurisdiccionales que apuntaban a la certificación de calidad, como lo eran la primera sala civil y el juzgado décimo segundo de lo civil del primer distrito judicial.

En este informe el Lic. Guerrero Gutiérrez expresó que el sistema de calidad en las áreas administrativa, resulta ser de suma importancia ya que la adecuación de la norma ISO permite que los operadores de justicia y servidores públicos sean evaluados de acuerdo al servicio ofertado a la comunidad, también recalco que la certificación ISO visibilizaría a la institución por ser parte de un sistema de estandarización de orden mundial.

Dando continuidad al informe, también hay que destacar algunos beneficios que trae consigo la aplicación de la norma ISO 9001, entre ellos están:

Lograr un trabajo más organizado al pie de una metodología. 
La labor se desempeña de manera eficiente.

Fomenta la competitividad en el ámbito laboral.

Se detectan indicadores sobre las acciones contrarias a la ley.

Implementa acciones correctivas y preventivas.

Se promueve la capacitación de los actores sobre las políticas que debe contener un sistema de calidad.

Disminuye el número de quejas

Desarrolla altos niveles en el rendimiento del personal.

Posterior a todo el proceso de análisis, se elaboró un manual de calidad con instrucciones sobre el trabajo y registro de la calidad, procedimientos generales, instrucciones de trabajo y registro de la calidad, que fueron utilizados para evaluar el desempeño de otras entidades jurisdiccionales (TJS, 2003).

\section{Norma ISO 9001 adoptada por la Procuraduría Federal del Consumidor (PROFECO)}

La Profeco es la institución encargada de la protección del consumidor, así como de difundir el consumo inteligente. Ambas actividades cuentan con un área de laboratorio para su operación, este sitio es donde la institución realiza diferentes estudios a la diversidad de productos que existen en el mercado, los resultados de estas pruebas son publicados en diferentes medios de comunicación, en donde se enuncia si el producto cumple con los estándares de calidad (David, 2016).

\section{Cuadro 3. Criterios de calidad de Profeco.}

\begin{tabular}{l|l} 
Sistema de gestión de calidad & $\begin{array}{l}\text { específicas metodologías y sistemas de control de } \\
\text { calidad. } \\
\text { Supervisa las actividades de Profeco y la vigilancia y } \\
\text { cumplimiento SGC (Sistema de Gestión de Calidad). } \\
\text { Cumple en planear la manera en que va a asignar los } \\
\text { recursos, tomando en cuenta las recomendaciones del } \\
\text { cliente. } \\
\text { Se define los requerimientos del producto y del clien- } \\
\text { Gestión de los recursos } \\
\text { te, así como del proceso para cada servicio. } \\
\text { El SGC es monitoreado de manera completa por } \\
\text { satisfacción del cliente, análisis de tendencias, expe- } \\
\text { dientes de proveedores. }\end{array}$ \\
\hline Medición de análisis y mejora
\end{tabular}

Fuente: Elaboración propia, (2020). 
El laboratorio nacional de la Profeco conto con la certificación NMX-ISO-9000-2000 y estuvo acreditado con NMX-ISO-17025-2005, también poseía un manual de calidad que cumplía con los requerimientos que establece el ISO 9000-2000, entre ellos los del cuadro 3.

En consideración a lo desarrollado en cuanto a la acreditación de estas instituciones del estado de Nuevo León, el Tribunal Superior de Justicia y la Profeco, ambas apuntaron a la calidad. El tribunal se enfocó en ofertar un servicio de calidad a los usuarios y a mejorar sus procesos internos de índole administrativos y la Profeco en ofertar y mantener la calidad en la variedad de los productos que son consumidos por la población. Ambas instituciones resultan ser de vital importancia para la sociedad al lograr la estandarización ISO de sus procesos, mejorando así su compromiso social con los usuarios o consumidores.

\section{Modelo europeo de gestión de calidad (EFQM)}

En 1988 fue creada la Fundación Europea para la Gestión de calidad (European foundation for quality management), que se encuentra constituida por 14 organizaciones europeas con un propósito en común, el cual era mejorar la calidad de las empresas en el referido continente. En 1922 la fundación creo el Modelo de Gestión de Calidad conocido mejor como EFQM (Diaz, 2016)

Carreón (2013) expresa que el modelo EFQM contiene nueve criterios que se subdividen en dos grandes grupos, el primero está destinado a los "agentes" que son los factores por los cuales se logran los fines y el segundo grupo son los "resultados" con los cuales se logran la organización; además el autor plantea el desglose de estos criterios:

\section{Agentes}

Liderazgo: Este criterio se refiere al compromiso y al estilo de la dirección que son ejercidos por la gerencia o quien esté a su cargo, también tiene relación con la calidad y el logro de los objetivos o misión de la organización, realzando entre ellos la satisfacción del cliente.

Politica estratégica: Es la dinámica en donde la organización implanta su misión y visión con la finalidad de desarrollar las estrategias, también describe el paso a paso de la implementación de políticas, planes, objetivos y procesos en donde se materializa la estrategia.

Persona: Se analiza la gestión y el desarrollo del conocimiento de las personas que son parte de la organización, esta acción permite evaluar el potencial de cada individuo o grupos. También se evalúa como se planifica las actividades con apoyo de la política y estrategias, además del eficaz funcionamiento de los procesos.

Recursos y alianzas: Este factor se refiere cuando la organización gestiona y planifica sus recursos internos, y la de sus colaboradores externos, con el objetivo de conocer como estos 
apoyan la política y la estrategia en la función eficaz de los procesos.

Procesos: Este factor es de suma importancia debido a que define la operatividad de la organización.

\section{Resultados}

Resultados en los clientes: Computa el grado de satisfacción y las necesidades de los clientes externos y la elección de medidas para evaluarlas.

Resultados en las personas: Se encarga de medir al igual que el anterior las necesidades y satisfacción de las personas, pero en este caso de los agentes internos o colaboradores.

Resultados en la sociedad: Este factor está relacionado con la responsabilidad social que tiene la organización desde esferas como la prevención de riesgos laborales, el impacto ambiental de los productos que distribuye y el comportamiento ético de la empresa.

Resultados clave: Asegura que la organización evalué el grado de cumplimiento de las metas y objetivos trazados u aquellos elementos que se han identificado como logros importantes, esto puede darse a corto o a largo plazo y esta enlazado directamente a aspectos financieros de la institución.

Por otro lado, existe también el Modelo Iberoamericano de Calidad creado por la fundación iberoamericana para la gestión de calidad (FUNDIBEQ), la cual estableció en el año 2000 la distinción del premio Iberoamericano ala Calidad. Este modelo tiene una gran relación con el modelo EFQM en cuanto a su estructura, clasificación y los criterios que aborda, pero también difiere en la puntuación cuantitativa de alguno de los criterios (Carreon, 2013)

\section{Modelo del premio nacional de calidad mexicano (PNC)}

Este modelo fue creado a finales de los años 80 por un grupo de expertos que tenían como propósito impulsar la mejora continua de las instituciones u organizaciones mexicanas. El eje principal de PNC se trata de reforzar el valor superior de los clientes y los consumidores atreves de una mejora continua de los sistemas, procesos, productos y servicios.

Dentro de los propósitos del PNC están (FPNC, 2016):

Estimular el establecimiento de los procesos integrales

Promover la productividad y la calidad en los productos, servicios y procesos

Promover el uso del modelo nacional para la calidad total en las organizaciones mexicanas.

Fomentar las exportaciones en base a la calidad

Promover visibilidad de las empresas

Ofrecer una herramienta útil de diagnóstico y mejora continua

Este modelo a diferencia del EFQM se subdivide en tres bloques de acuerdo al ciclo Deming 
y además evalúa 8 criterios, que a continuación se pueden apreciar en la siguiente gráfica:

Gráficas 2. Criterios de evaluación de la Calidad - PNC

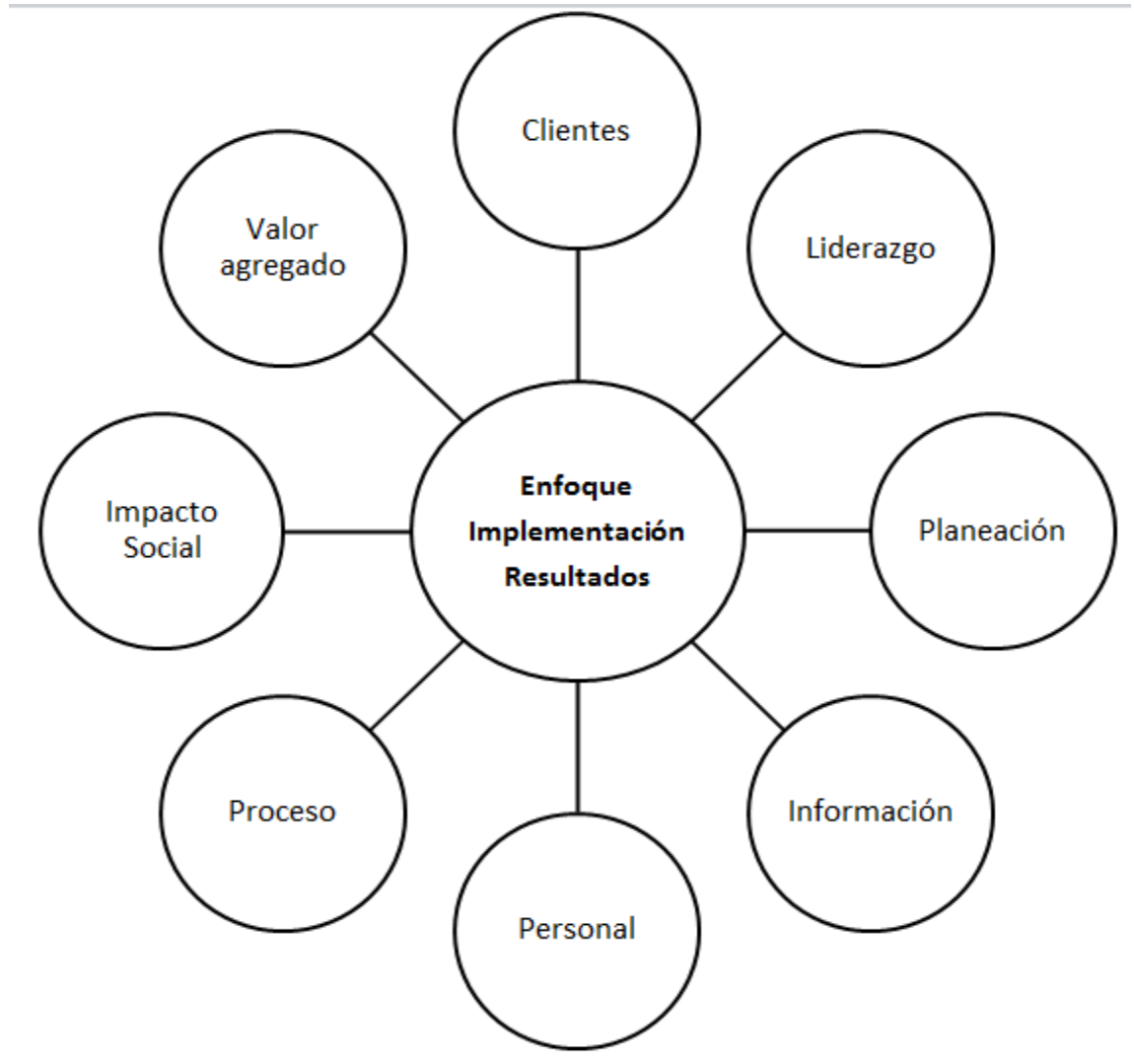

Fuente: Elaboración propia, (2020).

\section{Evaluación de calidad del proceso de mediación}

Se puede observar que los modelos de evaluación que anteceden han sido implementados en distintas instituciones dedicadas a diferentes tópicos como lo son: la salud, el comercio, el medio ambiente, la seguridad en los centros de trabajos, los sistemas de justicia entre otros. Sin embargo, hasta el momento en la mayoría de países no hay una aplicación centralizada ni estandarizada de un sistema que evalué y procuré la calidad del servicio de mediación (Gonzalez Ramirez, 2018).

Es relevante mencionar que existen diferentes teorías donde se observa que el servicio de mediación puede ser evaluado de diferentes enfoques:

La primera está basada en lo que considera el consumidor en relación al servicio (usuario en el proceso de mediación), de lo cual se engloban las calidades técnicas que consisten en la acreditación profesional y formación del mediador, y la segunda se enfoca cuando el consumidor 
juzga el servicio después de haber sido ofertado, siendo este último un factor difícil de medir en la mediación, en vista que posterior a la realización del acuerdo existen muchos factores externos que pueden incidir en la apreciación de las partes, en cuanto al servicio ofertado y la calidad del mismo (Lehtinen, \& Lehtinen, 1982).

\section{Evaluación de la mediación en base a los resultados}

Pruitt \& Carnevale (1993) plantean dos criterios de evaluación de la eficacia de la mediación. Como primer criterio esta la evaluación a corto plazo, la cual corresponde a enunciados que determinan una buena mediación como, por ejemplo:

1. El sentimiento expresado por partes de la mediación, referido a la satisfacción de la misma.

2. La voluntad de cumplir los acuerdos alcanzados.

3. La percepción de la eficacia de la mediación, esto implica funcionamiento, rapidez, economía etc.

4. El cumplimiento de lo acordado.

Se deberá tener en cuenta que estos criterios deben de ser evaluados al finalizar la mediación.

Por otra parte, están los criterios de evaluación a largo plazo, los cuales se basan en las siguientes actividades:

1. El grado de cumplimiento de los acuerdos

2. Las mejoras de las relaciones entre las partes.

El conjunto de criterios de evaluación antes relacionados sirven para diagnosticar si se tiene como resultado una mediación "exitosa" sin embargo hay que delimitar cuales son los factores que determinan la eficacia del método (Gonzalo Serrano, 2008).

\section{Evaluación de la mediación en base a la percepción del servicio ofertado}

La percepción del usuario para evaluar a la mediación como un servicio, es otro de los enfoques por los cuales se puede abonar a la calidad del proceso mediación.

Para Díaz Álvarez (2016) la evaluación de la mediación puede ser valorada desde diferentes factores endógenos que determinan la calidad, es por ellos que plantea en su investigación titulada "Mediación Calidad" seis características que son base para la evaluación del este servicio; en las cuales encontramos:

Características físicas de las instalaciones

Habilidad del centro de mediación, servicio promedio y confiable

Disponibilidad de servicio al usuario

Conocimiento, cortesía y confianza por parte del personal hacia el usuario 
Cuidado y atención personalizada al usuario

Valor de la mediación en la sociedad (intangibles de la mediación)

Todas estas características estuvieron enfocadas en evaluar la calidad del servicio de mediación que se oferta a la sociedad por parte de los diferentes centros del estado de Nuevo León tanto en el ámbito público como privado. Para llevar a cabo la evaluación referida, Álvarez (2016) aplico el modelo que se describe a continuación.

Modelo service quality (SERVQUAL)

Ilustración 1. Cronología de hechos internacionales, teorías de calidad y modelos de evaluación

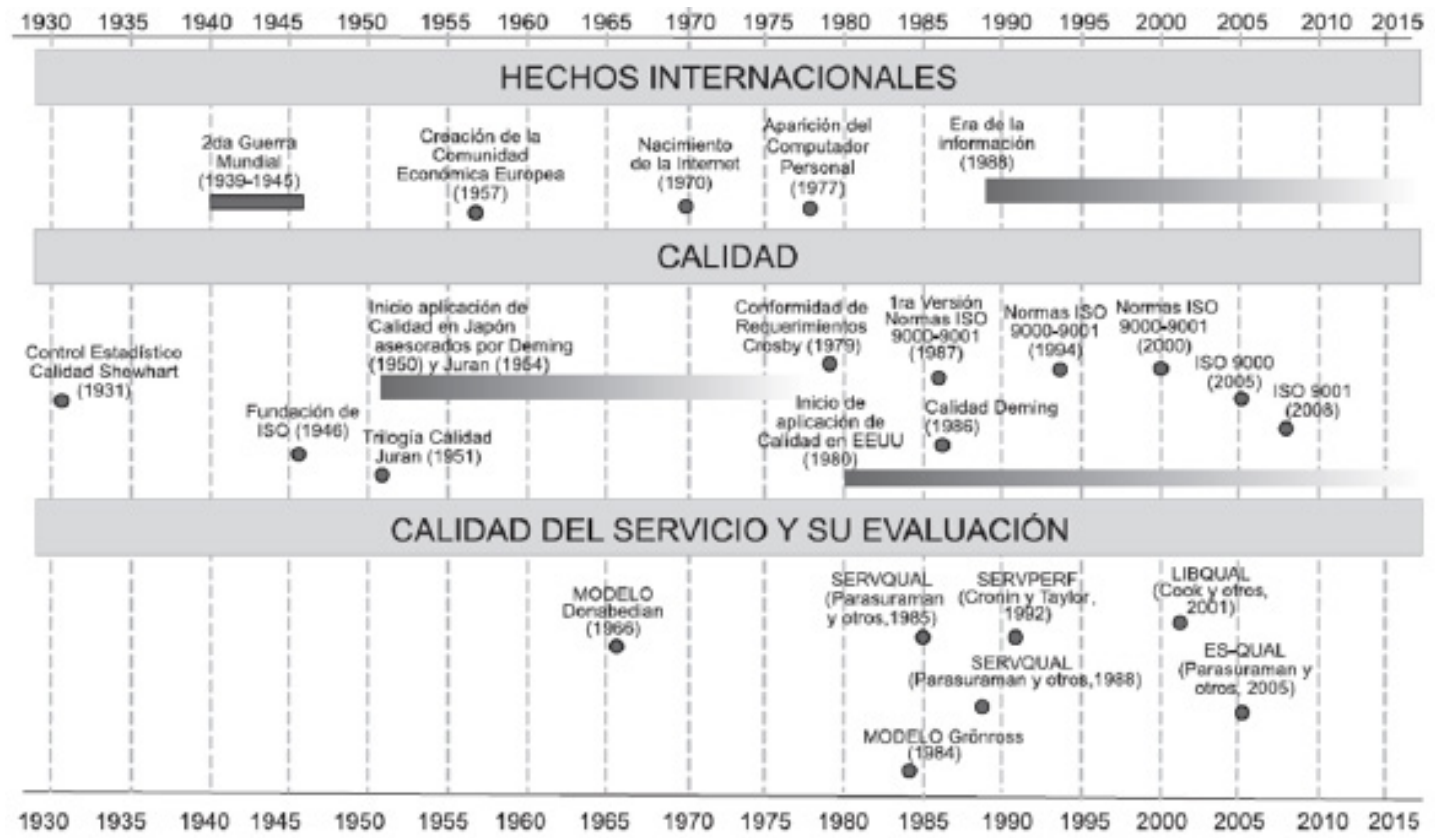

Fuente: (Torrez , Maritza, Vasquez, \& Carmen, 2015)

En la figura que antecede se muestra una cronología de los principales modelos de evaluación de la calidad, también se destacan hechos internacionales y algunas teorías que contribuyen a la evaluación de la calidad, en las cuales se puede observar algunos de los modelos que ya se comentaron en este artículo. Por otro lado se puede apreciar el surgimiento del modelo SERVQUAL que deviene históricamente de la escuela americana de marketing y fue desarrollado por los investigadores Parasuraman, Berry y Zeithaml en 1985 y posteriormente 1988 por medio del análisis factorial se simplifico y albergo la evaluación de los servicios bajo estas cinco dimensiones (Torrez, Maritza, Vasquez, \& Carmen, 2015): 
a. Empatía: Muestra de interés a nivel de intención individualizada que ofrece la institución al usuario.

b. Fiabilidad: Habilidad para ejecutar el servicio prometido de forma fiable y cuidadosa.

c. Seguridad: Conocimiento y atención de los empleados y su habilidad para inspirar confianza.

d. Capacidad de respuesta: Disposición para ayudar a los usuarios y para presentarles un servicio rápido.

e. Elementos tangibles: Apariencia física de las instalaciones físicas, material, equipos y personal.

Se puede apreciar claramente que Díaz Álvarez (2016) en su investigación titulada “Mediación Calidad“" adecuo las dimensiones que platea el modelo SERVQUAL a la evaluación del servicio de mediación, utilizando este prestigioso gestor de calidad para medir las diferentes fases del proceso con la finalidad de procurar la calidad y eficiencia en el trámite de mediación. Por lo tanto, dicha investigación resulta ser la pionera en estandarizar un modelo de calidad dedicado a evaluar la satisfacción de los usuarios en el ámbito de la justicia alternativa, ya que este modelo comúnmente se aplica en esferas como a salud, la educación, industria, hotelería y otro sin números de servicios enfocados en procurar la calidad de los procesos.

\section{Evaluación de la mediación con base a la capacidad y competencias del mediador}

Para Aguilar (1994) un elemento determinante para contribuir a la calidad del proceso de mediación está enfocado en las competencias y capacidades del mediador. Partiendo de este enfoque se constituyó en Chile una mirada sistemática a la práctica del mediador, que tuvo como propósito el mejoramiento continuo de las prácticas profesionales por medio de la implementación de una metodología cualitativa y cuantitativa que tuvo como base la observación del mediador bajo la evaluación y medición de un sistema llamado evaluación de calidad del proceso de mediación familiar (ECAME).

\section{Instrumento de medición de calidad ECAME}

En Chile a partir del año 2011 se han venido implementado un sistema de supervisión entre pares que tiene como finalidad establecer una modalidad de aprendizaje-acción apegado a los lineamientos de la investigación experimental, esto consistió en el aprovechamiento de los conocimientos adquiridos de la práctica y la experiencia, que tuvo por objeto mejorar los sistemas de servicios de mediación existentes y de igual forma contribuir a la calidad del proceso (Gonzalez Ramirez, 2018)

Dentro de los antecedentes metodológicos del referido sistema se constituyó un estudio ini- 
cial denominado “Auditoria de Calidad de los Servicios de Mediación Familiar” realizado por UCEN, que de acuerdo a Muños (2015) perseguía las siguientes disposiciones:

Diseñar un sistema nacional de gestión de supervisiones clínicas para los mediadores.

Implementar la aplicación del ECAME de supervisión.

Sistematizar cualitativamente y cuantitativamente los resultados.

Generar un proceso de retroalimentación y perfeccionamiento del desempeño profesional, estableciendo criterios de calidad compartidos.

Este sistema en la actualidad cuenta con un proceso que costa de cinco etapas. La primera se enfoca en la supervisión y observación del proceso de mediación, en esta fase se utiliza la metodología de la video grabación para obtener un mejor análisis, en segundo puesto se sitúa la autoevaluación del mediador observado, para que posteriormente de inicio la tercera fase del proceso que consiste en la retroalimentación que debe de ejercer el supervisor al mediador evaluado. El cuarto paso se trata de la elaboración de un informe y la asignación del puntaje al mediador de conformidad a una escala enfocada en los diferentes criterios de evaluación del servicio y sobre todo de la calidad del mismo, y por último en la etapa número cinco se concentra la capacitación remedial de aquellos factores en donde se encontró un déficit que conlleva a la elaboración de manuales de buenas prácticas para fortalecer los procesos de mediación (Gonzalez Ramirez, 2018).

\section{3.- MÉTODO}

\section{Diseño}

Esta investigación posee un enfoque meramente cualitativo, en vista que no se trata de una mera especulación, interpretación o reflexión del suscrito investigador, por lo contrario, se basa en comprobar la existencia del fenómeno de estudio que da origen a la pregunta de investigación y por ende la hipótesis propuesta.

Entre otras características el estudio parte de un diseño no experimental debido a que no se manipula ninguna de las variables propuestas, es decir se trata de una investigación en la cual no se aplica la variación de las variables. También posee un enfoque exploratorio, en vista que la problemática planteada resulta ser un fenómeno del cual existe escasa literatura. Cabe destacar que la investigación exploratoria analiza a las variables o factores que podrían estar relacionados a la problemática y finaliza cuando ya se obtiene una idea clara y relevante de las variables y cuando se justifica que ya hay suficiente información sobre el tema (Abreu, 2012)

\section{Instrumento}

Con el objetivo de recopilar la información que contiene el marco teórico, se llevó a cabo una 
exhaustiva revisión de la bibliografía relacionada al sustento de las variables de investigación, por medio del ejerció de la lectura comprensiva en donde se analizaron con un enfoque critico las treinta y una referencias bibliográficas.

\section{Procedimiento}

Primeramente, se realizó un análisis compresivo de las fuentes que están meramente relacionadas a la problemática de estudio, como resultado se dedujo que existía escasa información sobre la aplicación de los modelos de evaluación de calidad en Centros que ofertan el servicio de mediación en México. Por tal razón se realizó un análisis comparado del fenómeno de lo cual se obtuvo información derivada de investigaciones académicas y posterior se realizaron conclusiones y recomendaciones al estudio.

\section{4.- CONCLUSIONES}

En los apartados que anteceden se desarrolló de manera clara todo lo relacionado a la calidad en general y a los tipos de modelos que la evalúan. También se llevó acabo un análisis de algunas esferas en las que se han aplicado los diferentes sistemas de evaluación de la calidad. En base a lo dicho podemos concluir lo siguiente:

La calidad es un concepto que trasciende más allá de una actividad en específico, puede ser considerada como una aptitud, cultura o estilo de vida de un individuo que tiene como fin alcanzar los máximos estándares de excelencia.

La calidad puede ser analizada desde el enfoque del producto, la calidad del servicio prestado o las fases de un proceso que conllevan a estándares de excelencia.

La mediación debe ser considerada un servicio en vista que cuenta con todos los elementos esenciales del mismo.

Los modelos de calidad ISO 9000, 9001, EFQM y el PNC concuerdan en los siguientes criterios:

Todos toman en cuenta la satisfacción del cliente.

Todos concuerdan que el proceso es un factor clave para la operatividad de las organizaciones.

Los tres modelos toman en cuenta la eficiencia, capacitación y evaluación del personal.

Los modelos de calidad ISO 9000, 9001, EFQM Y PNC no han sido implementados en evaluar la calidad del servicio de mediación.

Los modelos de evaluación como SERVQUAL y ECAME ya fueron implementados en evaluar los servicios de mediación con el propósito de mejorar la calidad del servicio ofertado. Por tanto podemos concluir que en México existe la necesidad de implementar los modelos 
de evaluación de calidad nacionales e internacionales, con el propósito de garantizar que el servicio de mediación ofertado, este avalado por certificaciones de alto prestigio que generan confianza, de igual forma se recomienda que los centros de mediación públicos y privados, implementen sistemas de calidad de manera interna con el objetivo de efectuar evaluaciones periódicas que fomenten la mejora constante del servicio de mediación.

\section{REFERENCIAS}

Baganha, Abreu, J. L. (2012). Hipótesis, Método \& Diseño de Investigación. International Journal of Good Conscience, 7(2), 187-197.

Aenor . (2002). ISO 9001 para la pequeña empresa recomendaciones del comite ISO/TC. Asociacion española de normalizacion y certificacion.

Aguilar Ibáñez, M. J. (1994). Introducción a la supervisión. Buenos Aires: Lumen.

Barraza, M. F. (2007). La Filosofía de Mejora Continua e Innovación. Mexico.

Carreon, A. (2013). El model EFQM mas alla de ISO 9000. Revista informativa basica, 1(1), 26-70.

Castañeda Salas. (2019). Pendiente. Mexico, Nuevo Leon: UANL.

David. (28 de Abril de 2016). Laboratorios Profeco- Sistema de gestion y calidad. Monterrey, Nuevo Leon, Mexico. Obtenido de http://gestiondcalidad.blogspot.com/2016/04/ laboratorio-profeco-sistema-de-gestion.html

Diaz Alvarez, R. (2016). La calidad de la mediacion en Nuevo Leon Mexico. Murcia, España.

Druker, P. (1990). El ejecutivo eficaz. Buenos Aires: Sudamericana.

Duque Oliva, E. J. (2005). Revisión del concepto de calidad del servicio y sus modelos de medición. INNOVAR. Revista de Ciencias Administrativas y Sociales, 15(25), 64-80. Obtenido de http://www.redalyc.org/articulo.oa?id=81802505

Estado, C. d. (13 de Enero de 2017). Ley de Mecanismos alternos para la solución de controversias para el estado de Nuevo León. Ley.

FPNC. (2016). Premio Nacional de Calidad. Obtenido de http://www.pnc.org.mx/el-premionacional-de-calidad/

Gonzalez Ramirez, I. X. (2018). La calidad de la mediacion familiar en chile. Revista de deRecho PRivado(35), 369-390. 
Gonzalo Serrano. (Marzo de 2008). Eficacia y Mediacion Familiar. 53.

Imai, M. (1998). Cómo implementar el kaizen. Bogota: McGraw-Hill Interamericana.

Ishikawa, K. (1986). ¿Qué es control total de la. Bogota: Norma.

Lechuga, J., Cazallo , A., Olivero, E., \& Estruen, D. (2017). Diagnóstico de las Pymes Colombianas de los sectores de manufactura y comercio, en Proceso de Internacionalización de las Pymes. Proceso de internacionalizacion de en las pymes. Colombia: Universidad de Simon Bolivar.

Lehtinen,, U., \& Lehtinen,, J. R. (1982). Service quality: A study of quality dimensions. The Services Industries Journal, 2(3). Obtenido de https://technopreneurship.piles.wordpress. $\mathrm{com} / . . . / \mathrm{dra}$

Lovelock, C. H. (1983). Classifying Services to Gain Strategic Marketing Insights. Journal of Marketing, 9-20.

Merlo Vega, J. A. (2011). Innovacion educativa en la enseñanza tecnicas. Boletin de la asociacion austriana de bibliotecarios .

Miranda Gonzalez, A. C. (2007). Introducción a la gestión de la Calidad. Madrid: Delta Publicaciones.

Muños Leon , F. (2015). ¿Hacia la academización de las facultades de derecho en Chile? Un análisis teórico y comparado del conlicto de las profesiones. Revista de derecho, 81-95.

Novel Marti, G. (s.f.). El mediador y el manejo de emociones. Barcelona.

Pruitt, D., \& Carnevale, P. (1993). Negotiation in Social Conflicts.

RAE. (2019). https://www.rae.es/. Obtenido de https://www.rae.es/.

Santana Rodríguez, G., \& Rodriguez-Mateo, H. (2014). El perfil de la eficacia del mediador desde la perspectiva de la negociacion. International Journal of Developmental and Educational Psychology, 237-244.

SE. (2016). Normalizacion Gobierno. Obtenido de www.2006-2012.economia.gob.mex/ comunidad-negocio/normalizacion

Sun, T. (2009). El arte de la guerra. Mexico: Gaia Ediciones.

Tafolla, H. (2000). Instituto autonomo de Mexico. Obtenido de Estandarizacion y globaliazion: www.itan.com.mx 
TJS. (2003). Tribunal Superior de Justicia del Estado de Nuevo Leon, Nuevo Leon.

Torrez , S., Maritza, Vasquez, S., \& C. L. (2015). Modelos de evaluación de la calidad del servicio: caracterización y análisis. 18(35), 57-76. Obtenido de http://www.redalyc.org/ articulo.oa? $\mathrm{id}=88043199005$

Zeithaml, V., Berry, L., \& Parasuraman, A. (1988). Communication and Control Processes in Delivery of Service Quality. Journal of Marketing, 35-48. 